> Les maladies héréditaires du métabolisme ont acquis une place de plus en plus importante dans la pathologie pédiatrique. Leur nombre ne cesse d'augmenter au fur et à mesure de la progression des connaissances en biologie cellulaire et des progrès techniques d'investigation. Nous traiterons ici de trois maladies métaboliques que l'imagerie fonctionnelle et la spectroscopie IRM ont permis d'identifier. II s'agit des déficits en créatine traitables par l'administration de créatine et les défauts du métabolisme des polyols qui ouvrent le champ sur de nouveaux déficits enzymatiques responsables de présentations cliniques très variées. Nous aborderons également les hyperinsulinismes du jeune enfant dont le diagnostic et la prise en charge ont été récemment transformées par l'utilisation de la [18F]-fluoro-L-DOPA en tomographie par émission de positons. <

\section{Nouveautés radiologiques dans le dépistage et le diagnostic des erreurs innées du métabolisme}
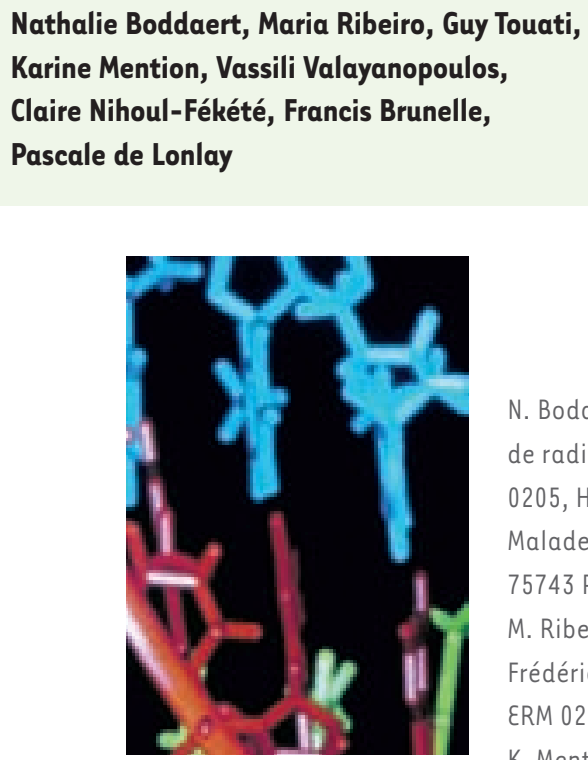

N. Boddaert, F. Brunelle : Service de radiologie pédiatrique, $\varepsilon R M$ 0205, Hôpital Necker EnfantsMalades, 149, rue de Sèvres, 75743 Paris Cedex 15, France. M. Ribeiro : Service Hospitalier Frédéric Joliot, DSV, DRM, CEA, ERM 0205, Orsay, France.

K. Mention, V. Valayanopoulos, P. de Lonlay, G. Touati : Service

un traitement dans un En 30 ans, les maladies héréditaires du métabolisme ont acquis une place de plus en plus importante dans la pathologie pédiatrique. Leur nombre ne cesse d'augmenter au fur et à mesure de la progression des connaissances en biologie cellulaire et des progrès techniques d'investigation [1, 2]. Les maladies métaboliques résultent le plus souvent d'un déficit enzymatique sur l'une des nombreuses voies métaboliques, dérivées des glucides, protides ou acides gras. Ces voies métaboliques permettent la transformation biochimique d'un composé en un autre, et par conséquent la dégradation et la synthèse de molécules nécessaires à l'organisme. Un déficit de l'une des enzymes impliquées dans ces voies métaboliques entraîne l'absence d'un composé situé en aval de la voie biochimique bloquée et/ou l'accumulation d'un composé situé en amont du déficit enzymatique. Ce composé, dès lors qu'il est présent dans le liquide céphalorachidien, peut être dans certains cas identifié grâce à l'imagerie cérébrale IRM avec spectroscopie. Le diagnostic des maladies métaboliques est important car, en dehors du conseil génétique, il permet

Article reçu le 8 septembre 2005, accepté le 19 septembre 2005. grand nombre de cas. Ce traitement consiste en un traitement diététique (suppression d'un composé toxique pour le patient, apport calorique, évitement du jeûne selon les cas), l'apport d'un cofacteur qui ne peut être synthétisé ou qui peut pallier le déficit enzymatique, ou encore une enzymothérapie apportant l'enzyme manquante [3].

Les maladies héréditaires du métabolisme, principalement de transmission autosomique récessive, mais aussi récessive ou dominante liée à l' $X$, autosomique dominante ou encore de transmission mitochondriale, peuvent être classées en trois groupes en fonction de leur mécanisme pathogène: (1) les maladies d'intoxication protéiques ou des sucres; (2) les déficits énergétiques et anomalies de synthèse ou de catabolisme des molécules complexes incluant les maladies lysosomales, les maladies peroxysomales, les anomalies de 
glycosylation des protéines (CDG) ; (3) les déficits héréditaires de la synthèse endogène du cholestérol. La compréhension de cette classification schématique aide au diagnostic lorsqu'une maladie métabolique est évoquée.

Nous traiterons de trois maladies métaboliques que la spectroscopie et l'imagerie fonctionnelle ont permis d'identifier. II s'agit des déficits en créatine traitables par l'administration de créatine et les défauts du métabolisme des polyols qui ouvrent le champ sur de nouveaux déficits enzymatiques responsables de présentations cliniques très variables. Nous aborderons également les hyperinsulinismes du jeune enfant dont le diagnostic et la prise en charge ont été récemment transformées par I'utilisation de la [18F]-fluoro-L-DOPA en tomographie par émission de positons (TEP).

\section{Déficit de synthèse de la créatine}

Déficits primaires de synthèse de la créatine : déficits en AGAT, GAMT, ou récepteur de la créatine

La créatine est synthétisée dans le foie et le pancréas à partir de l'arginine (Figure 1). Elle est phosphorylée par la créatine kinase et donne aux cellules cérébrales et musculaires ses phosphates pour la synthèse de I'ATP. Les déficits en guanidinoacétate méthyltransférase (GAMT), arginine glycine amidinotransférase (AGAT) et récepteur de la créatine (Xq28) sont responsables de retard mental [4]. Le retard mental peut être isolé ou associé à un autisme, à un syndrome extra-pyramidal ou à une épilepsie. La créatinine plasmatique dosée par le ionogramme sanguin peut être diminuée ou normale selon la méthode biochimique utilisée. Les dosages de guanidinoacétate et de créatine dans les urines et le plasma orientent le diagnostic de façon précise (Tableau I).

Biochimie

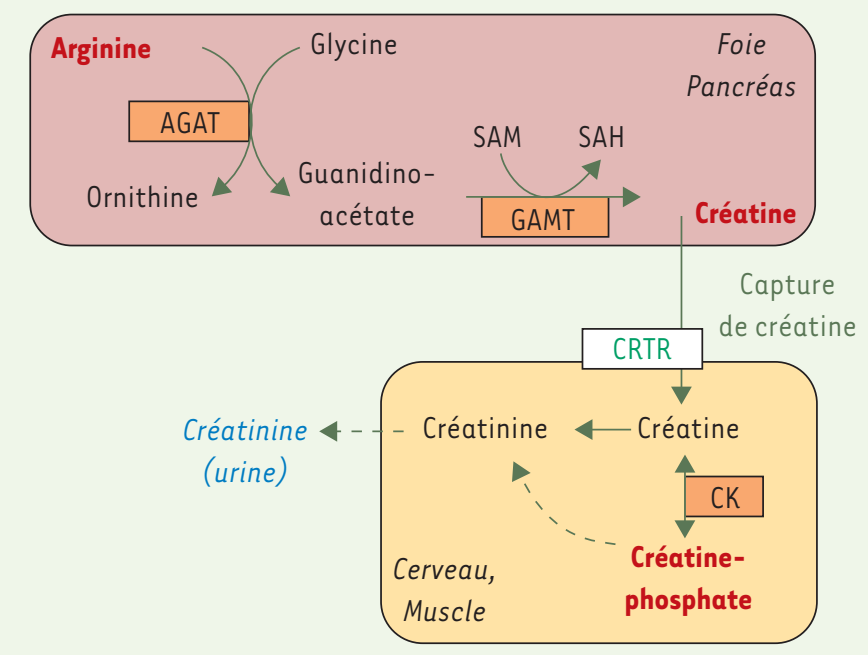

Figure 1. Synthèse de la créatine dans le foie et le pancréas à partir de l'arginine. AGAT: arginine glycine amidinotransférase; GAMT: guanidinoacétate méthyltransférase; SAM : S-adénosyl méthionine; SAH : S-adénosyl homocystéine ; CRTR : transporteur de la créatinine ; CK : créatine kinase.
Les dosages enzymatiques se font à partir de lymphocytes, tissu hépatique ou fibroblastes. L'imagerie anatomique cérébrale est normale, le plus souvent, ou peut montrer comme cela a été décrit une anomalie de signal des globi pallidi $[5,6]$. La spectroscopie IRM, quant à elle, montre l'absence de pic de créatine dans tous les cas, même en cas de défaut du récepteur. Ainsi la spectroscopie IRM, qui devrait idéalement être réalisée avec toute IRM cérébrale dans l'investigation étiologique d'un retard psychomoteur, permet le diagnostic des déficits en créatine. Dans notre expérience, nous avons diagnostiqué un déficit en créatine (GAMT) grâce à la spectroscopique IRM chez un enfant présentant un trouble du comportement de type autistique avec retard psychomoteur et une épilepsie (Figure 2B).

Une amélioration de l'état neurologique est observée avec l'administration orale de créatine, (350 mg/kg/ jour-2 $\mathrm{g} / \mathrm{kg} /$ jour) dans les déficits en GAMT et AGAT. Un régime pauvre en arginine et riche en ornithine est également prescrit dans le déficit en GAMT du fait d'une possible toxicité cérébrale du guanidinoacétate. Cependant, l'administration de créatine peut parfois suffire à diminuer le taux de guanidinoacétate car la créatine exerce un contrôle négatif sur l'enzyme AGAT.

\section{Déficits secondaires de synthèse de la créatine}

Des déficits secondaires de la créatine sont parfois observés dans d'autres maladies métaboliques s'accompagnant d'une hyperornithinémie, comme les déficits en ornithine delta aminotransférase, responsables d'une atrophie gyrée et d'une cécité à l'âge adulte (déficit en ornithine aminotransférase, OAT). En effet l'ornithine exerce un contrôle négatif sur l'enzyme AGAT et par conséquent sur la synthèse de la créatine $[7,8]$. Le retard mental et les anomalies musculaires qui accompagnent dans un certain nombre de cas l'atrophie gyrée pourraient être liés au déficit secondaire en créatine. L'IRM anatomique cérébrale est le plus souvent normale ou montre une anomalie de la substance blanche non spécifique, mais la spectroscopie IRM montre une diminution

\begin{tabular}{llll}
\hline & GAMT & AGAT & R \\
Créatine & $\downarrow$ & $\downarrow$ & $\uparrow$ \\
Guanidinoacétate & $\uparrow$ & $\downarrow$ & $\mathrm{N}$ \\
\hline
\end{tabular}

Tableau I. Dosages de créatine et guanidinoacétate (plasma, urines) dans les déficits en GAMT (guanidinoacétate méthyltransférase), AGAT (arginine glycine amidinotransférase) et récepteur de la créatine $\left(\chi_{q} 28\right)(R) . \downarrow$ : dosage diminué ; $\uparrow$ : dosage augmenté ; $\mathrm{N}$ : dosage normal. 
nette du pic de créatine [9]. La Figure 2C illustre le cas d'un enfant présentant un déficit en OAT avec retard mental. Dans notre expérience, le pic de créatine dans les déficits secondaires en créatine est diminué (Figure 2C) mais il est moins diminué que dans les déficits primaires en créatine (Figure $2 B$ ).

\section{Anomalies des polyols}

Les déficits héréditaires du métabolisme des polyols, métabolites sur la voie des pentoses, représentent de nouvelles maladies métaboliques décrites il y a moins de trois ans et probablement responsables de symptômes variés [10]. Nous allons décrire le déficit en ribose-5-phosphate isomérase où il existe un pic anormal à la spectroscopie IRM et le déficit en transaldolase qui présente une spectroscopie normale.

Le diagnostic du déficit en ribose-5-phosphate isomérase est évoqué devant l'accumulation de ribitol, arabitol et xylitol. Ce nouveau déficit a été mis en évidence par l'identification d'un pic anormal en spectroscopie IRM cérébrale correspondant à l'accumulation anormale de polyols cérébraux $[11,12]$. En effet, Huck et al. ont montré qu'il existait un pic anormal (3,6 et 3,8 ppm) de D-arabitol et ribitol sur la séquence de spectroscopie avec un temps d'écho (TE) court (20 ms) et également un pic anormal sur la séquence de spectroscopie à TElong (135 ms) (Figure 3). Une anomalie de signal majeure en séquence T2 et Flair au niveau de la substance blanche sus-tentorielle de façon bilatérale a également été mise en évidence [11, 13].

Le déficit en transaldolase, évoqué devant l'accumulation d'érythritol, arabitol et ribitol, a été impliqué dans une cirrhose hépatique de l'adulte puis dans une insuffisance hépatique néonatale [14, 15]. Nous avons identifié ce déficit chez des patients d'une même famille présentant une atteinte multiviscérale. Chez tous les patients, la grossesse a été marquée par un gain de poids excessif sans signe de toxémie gravidique, avec un placenta volumineux chez deux de ces patients. Des signes dysmorphiques, une atteinte hépatique avec hépatomégalie, une insuffisance hépatique et une fibrose, une splénomégalie, une anémie hémolytique, une thrombopénie, une atteinte rénale malformative et tubulaire, une atteinte cardiaque sont également présents. Aucun de ces patients n'a présenté d'atteinte neurologique significative alors que la transaldolase est présente dans plusieurs tissus différents, y compris l'encéphale. II n'existe pas d'accumulation de polyols dans le tissu cérébral de ces patients, comme il a été montré par la spectroscopie qui est normale et par un dosage normal des polyols dans le LCR, contrairement au déficit en ribose-5-phosphate isomérase. La toxicité des sucres phosphate accumulés pourrait rendre compte de l'atteinte hépatique et de la cirrhose, par analogie à la galactosémie dans laquelle un polyol (galactitol) et un sucre phosphate (galactose 1-phosphate) s'accumulent et entraînent une insuffisance hépatique. En revanche, l'atteinte multiviscérale est encore mal expliquée. Des travaux récents ont montré que la voie des polyols est une voie active pendant le premier trimestre de la grossesse, avec présence de fortes concentrations de certains de ces polyols dans le liquide amniotique [16].

Les deux principales fonctions métaboliques de cette voie sont la génération du NADPH sans production d'acide lactique et la formation
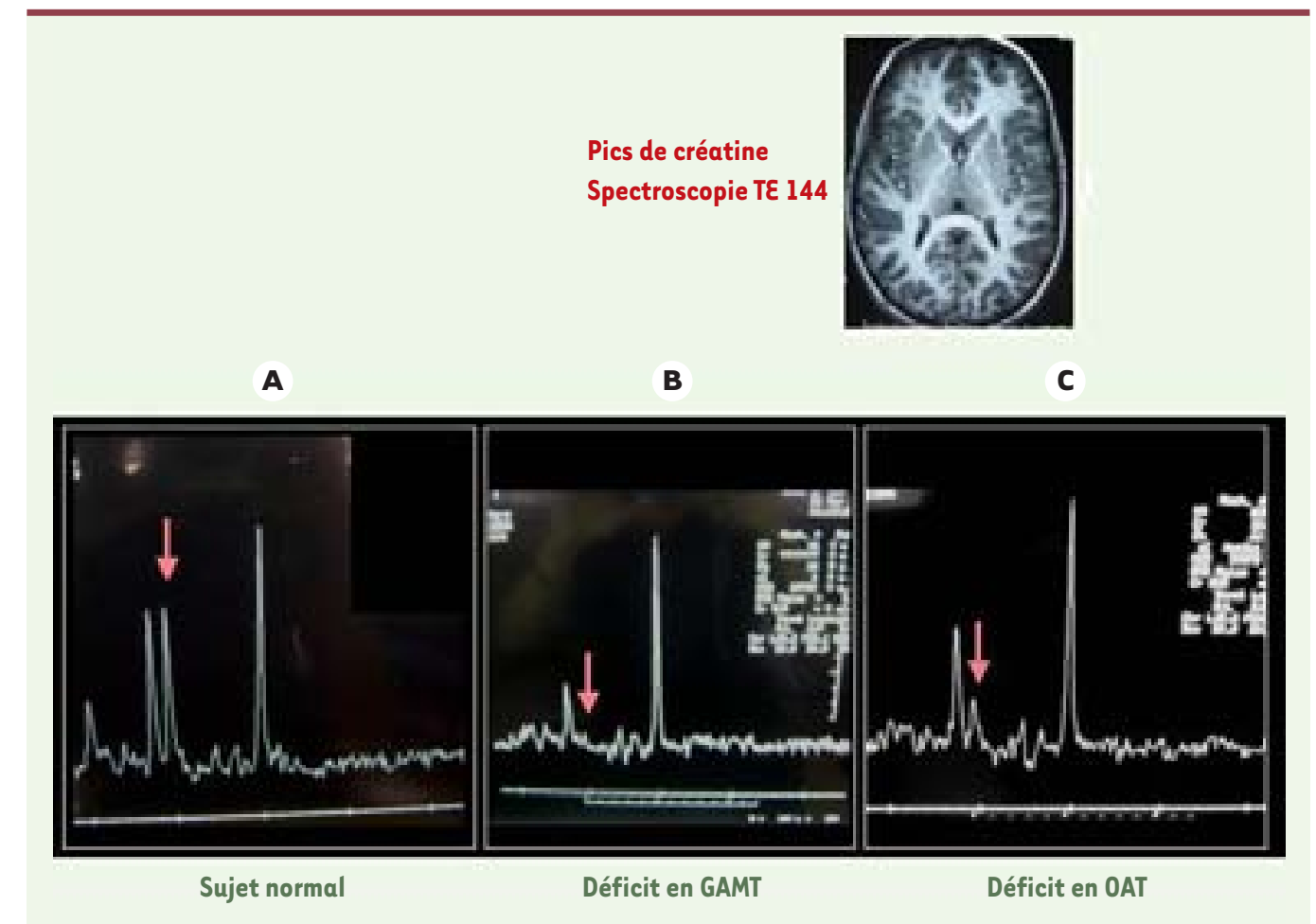

Figure 2. Spectroscopie IRM cérébrale dans les déficits en créatine. Les flèches verticales indiquent la position du pic de créatine. A. Sujet normal. B. Déficit primaire de synthèse de créatine par déficit en guanidinoacétate méthyltransférase (GAMT). C. Déficit secondaire de synthèse de créatine par déficit en ornithine aminotransférase (OAT) : le pic de créatine est moins diminué que dans le déficit primaire (en $B$ ). TE: temps d'écho. 
des pentoses phosphate nécessaires à la synthèse des acides nucléiques. Cette voie devient alors indispensable dans les tissus à croissance rapide comme les tissus embryonnaires. D'autre part, la génération du NADPH est indispensable aux voies de biosynthèse et est essentielle pour la régénération du glutathion réduit, le cofacteur de l'enzyme anti-oxydante glutathion peroxydase. L'importance de cette voie dans la protection de l'embryon contre les radicaux libres qui ont un potentiel tératogène a été démontrée. Ainsi, la diminution de l'activité de la glucose- 6 phosphate déshydrogénase aboutit à une incidence accrue de malformations congénitales sévères [17]. Les polyols peuvent également jouer un rôle dans l'homéostasie du fœtus, en particulier hydrique [18].

En résumé, la recherche d'un déficit en polyols par dosage des polyols urinaires doit faire partie des investigations devant tout anasarque fœto-placentaire avec oligoamnios chez les nouveau-nés ou nourrissons présentant une atteinte multiviscérale avec plus particulièrement une atteinte hépatique évoluant vers la cirrhose (déficit en transaldolase). La recherche d'un déficit en polyols par dosage des polyols urinaires et IRM cérébrale avec spectroscopie pour le dépistage des déficits en ribose-5-phosphate isomérase doit faire partie des investigations devant une atteinte neurologique, notamment devant une leucodystrophie.

\section{Hyperinsulinismes}

Les hyperinsulinismes sont la cause la plus fréquente d'hypoglycémie chez le jeune enfant. La sécrétion inappropriée d'insuline par le pan- créas est responsable d'hypoglycémies sévères entraînant un risque important de séquelles cérébrales graves [19]. La sévérité de l'hypoglycémie est la même quel que soit le type de lésion histologique du pancréas (formes focales ou diffuses). Cependant, le traitement chirurgical de l'hyperinsulinisme néonatal persistant diffère radicalement selon le type de lésion pancréatique [20-22]. En effet, les lésions focales peuvent bénéficier d'une résection chirurgicale localisée avec disparition des hypoglycémies (et guérison) alors que les formes diffuses résistantes au traitement médical nécessitent une pancréatectomie subtotale entraînant un diabète. Nous avons montré que les lésions focales sont dues à une mutation hétérozygote du gène du récepteur de la sulfonylurée (gène SURI) ou du gène Kir6.2 et d'une perte d'allèle de la région 11 pl5 limitée à la lésion $[23,24]$. La mutation des gènes SURI et Kir6.2 est toujours héritée du père, tandis que la perte d'allèle est toujours d'origine maternelle, faisant intervenir un mécanisme d'empreinte parentale. Les gènes SURI et Kir6.2 distants de $5 \mathrm{~kb}$ dans la région $11 \mathrm{pl} 15.1$ codent pour les deux sous-unités du canal potassique impliqué dans la sécrétion de l'insuline. Ainsi, les hypoglycémies hyperinsulinémiques sont liées à la perte d’hétérozygotie des gènes SURI ou Kir6.2, tandis que la lésion tumorale observée dans les formes focales est due à un déséquilibre entre les gènes suppresseurs de tumeur d'expression maternelle $(H 19, P 57)$ et les gènes de croissance d'expression paternelle (IGF2) situés en 1lp15.5. Ces gènes soumis à l'empreinte parentale sont déjà impliqués dans le développement d'autres tumeurs. Jusqu'à ces derniers temps, le seul examen réalisable pour différencier les formes focales des formes diffuses était le cathétérisme trans-hépatique (KTTH) qui permettait également de localiser les formes focales avant la chirurgie [25]. Cet examen permet de collecter du sang dans les différentes veines pancréatiques pour réaliser une cartographie de l'insulinosécrétion dans le pancréas. Cependant, cet examen est invasif, nécessite une anesthésie générale, et la mise en hypoglycémie obligatoire pendant toute la durée de l'examen et pendant les heures précédant l'examen. De plus, l'abord transhépatique est difficile et dépend de l'anatomie vasculaire veineuse très variable d'un enfant à l'autre. Seul le Professeur Brunelle, en Europe, était capable de le réaliser. Cet examen dépendant d'une seule personne, les nouveaux-nés hyperinsulinémiques en Europe à très fort ris- 
que d'hypoglycémies sévères attendaient des mois avant de pouvoir bénéficier de cet examen réalisé uniquement à I'hôpital Necker-Enfants Malades.

Le but de notre recherche était de trouver une méthode autre que le KTTH pour différencier les formes focales des formes diffuses. L'utilisation de la [18F]-fluoroL-DOPA en tomographie par émission de positons (TEP) a été testée et comparée au KTTH dans le diagnostic différentiel entre formes focales et formes diffuses d'hyperinsulinisme. En effet, la TEP avec utilisation de [18F]-fluoro-L-DOPA, déjà utilisé dans la maladie de Parkinson, a également été utilisée dans les tumeurs neuroendocrines [26]. La [18F]-fluoro-L-DOPA est convertie en [18F]-F-fluorodopamine par la DOPA décarboxylase. La production d'insuline est stimulée par le système parasympathique et inhibée par le système sympathique. Les foyers de production anormale d'insuline doivent être visualisés par un traceur qui se fixe sur les cellules productrices. Aussi, la [18F]-Ffluorodopamine, par ses propriétés de fixation sur les cellules neuroendocrines, doit fixer les lésions. L'utilisation de [18F]-fluoro-L-DOPA repose sur son analogie avec la L-DOPA, le précurseur physiologique de la dopamine. Ainsi, une fois injectée, la [18F]-fluoro-L-DOPA se comporte comme la L-DOPA. Elle est captée par un site spécifique des terminaisons dopaminergiques, puis est transformée en [18F]-F-fluorodopamine par la DOPA décarboxylase. Cet examen présente le grand avantage d'être une méthode non invasive qui peut être réalisée par d'autres équipes dans le monde. Une étude préliminaire nous a permis de s'assurer de la faisabilité de l'étude tant sur le plan de la réalisation pratique (réalisation simple ne nécessitant ni anesthésie générale ni hypoglycémie), que sur le plan diagnostique.

Ainsi, 15 enfants ( 11 garçons et 4 filles) avec un hyperinsulinisme néonatal, d'âge compris entre 1 et 14 mois, ont bénéficié d'un examen TEP corps entier avec une ECAT EXACT HR+ (CTI-Siemens) au CEA d'Orsay. Les enfants étaient à jeun ( 6 heures) et le traitement médical a été arrêté 72 heures avant l'examen. Un balayage thoraxabdomen (2 ou 3 segments d'une durée individuelle 2,5 minutes) a été réalisé 30 à 45 minutes après injection de [18F]-fluoro-L-Dopa (4MBq/kg). Quatre ou six balayages ont été effectués successivement. Tous ces enfants ont bénéficié d'un KTTH (méthode comparative/ gold standard). La TEP a permis une fixation localisée dans le pancréas pour les lésions focales et une fixation diffuse dans le pancréas pour les formes diffuses (Figure 4).

Les enfants avec suspicion d'une forme focale ont tous bénéficié d'une chirurgie pancréatique partielle, alors que seuls les enfants avec suspicion d'une forme diffuse résistante au traitement médical ont eu une pancréa- tectomie totale [19]. Parmi les 15 enfants initialement étudiés, une hyperfixation localisée de la [18F]-fluoroL-Dopa a été individualisée chez cinq enfants, au niveau de la tête ou du corps du pancréas, tandis qu'une distribution diffuse du radiotraceur a été observée dans le pancréas des dix autres patients (Figure 4). Les cinq enfants avec une hyperfixation localisée ont été soumis à résection chirurgicale partielle et ont été guéris. L’histologie per-opératoire a confirmé les résultats observés en TદP. Les résultats de cette étude suggèrent que la [18F]-fluoro-L-DOPA est capable de diagnostiquer et différencier les formes focales des formes diffuses d'hyperinsulinisme et de localiser les lésions focales des hyperinsulinismes néonataux. Depuis, la TEP a été réalisée chez 33 enfants sans réalisation préalable du KTH. Les suspicions de formes focales ont toutes été opérées alors que seules les suspicions de formes diffuses résistantes au traitement médical ont été opérées.

Malheureusement, le traceur utilisé n'étant pas commercialement disponible, l'accessibilité de la méthode aux cliniciens est très limitée. Cette limitation n'est pas exclusivement liée à un problème de réseau commercial. En effet, bien que le fluor-18 soit un isotope couramment utilisé en TEP, le mécanisme de production nécessaire au marquage de la Dopa et ce marquage lui-même suivent encore actuellement des procédures dont la complexité n'a rien à voir avec celles impliquées

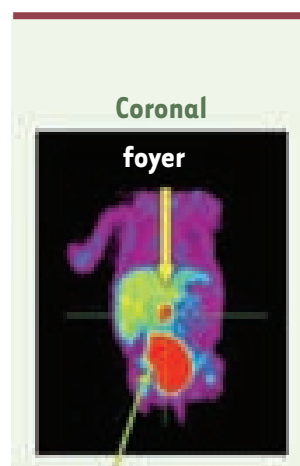

Vessie

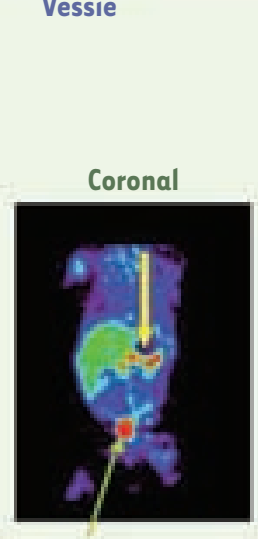

Vessie
Forme focale

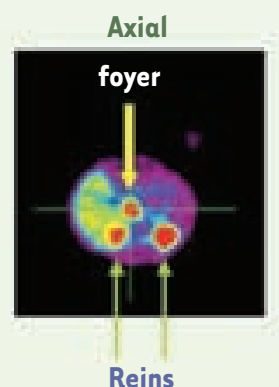

Reins

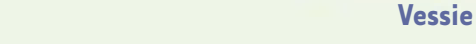

Forme diffuse

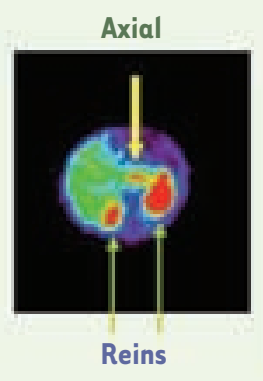

Reins

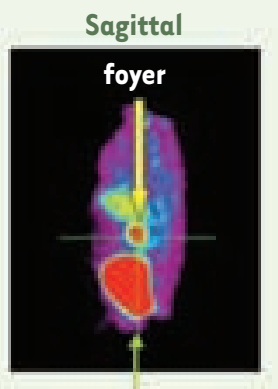

Vessie
Figure 4. Tomographie par émission de positons (TEP) avec utilisation de [18F]fluoro-L-DOPA dans les hyperinsulinismes. La TEP montre une fixation localisée dans le pancréas pour la forme focale et une fixation diffuse dans le pancréas pour la forme diffuse. 
dans le 18-fluoro-désoxyglucose. Cela explique le faible rendement de production de ce traceur et sont coût commercial potentiel, qui sont autant d'obstacles à sa diffusion.

\section{Conclusions}

Les progrès dans le diagnostic des maladies métaboliques ont été considérables durant ces cinq dernières années, notamment grâce aux progrès de la radiologie pédiatrique en spectroscopie IRM et en imagerie fonctionnelle. $\diamond$

\section{SUMMARY}

Radiological innovations in the diagnosis of the metabolic diseases : spectroscopy and cerebral MRI in creatine deficiencies, polyols anomalies, and metabolites on the pentose pathwaypositron emission tomography (PET) after injection of [18F]FluoroL-Dopa in congenital hyperinsulinism

New metabolic diseases are regularly identified by a genetic or biochemical approach. Indeed, the metabolic diseases result from an enzymatic block with accumulation of a metabolite upstream to the block and deficit of a metabolite downstream. The characterization of these abnormal metabolites by MRI spectroscopy permitted to identify the deficient enzyme in two new groups of diseases, creatine deficiencies and polyol anomalies. Creatine deficiency is implicated in unspecific mental retardation. A low peak of creatine at MRI spectroscopy is evocating of creatine deficiency which is treatable by creatine administration. Deficiency of synthesis of polyols, metabolites on the pentose pathway, represent new described metabolic diseases with variable symptoms including a neurological distress, liver disease, splenomegaly, cutis laxa and renal insufficiency. The deficit of ribose-5-phosphate isomerase, one of the enzymes whose diagnosis is evoked in front of the accumulation of ribitol, arabitol and xylitol leads to a leucodystrophy in adults. This new deficit was highlighted by the identification of an abnormal peak in cerebral MRI-spectroscopy corresponding to the abnormal accumulation of polyols in brain. Congenital hyperinsulinism (HI) is characterized by profound hypoglycaemia related to inappropriate insulin secretion. Focal and diffuse forms of hyperinsulinism share a similar clinical presentation but their treatment is dramatically different. Until recently, preoperative differential diagnosis was based on pancreatic venous sampling, an invasive and technically demanding technique. Positron emission tomography (PET) after injection of [18F]Fluoro-L-DOPA has been evaluated for the preoperative differentiation between focal and diffuse $\mathrm{HI}$, by imaging uptake of radiotracer and the conversion of [18F]Fluoro-L-DOPA into dopamine by DOPA decarboxylase. PET with [18F] Fluoro-L-DOPA has been validated as a reliable test to differentiate diffuse and focal $\mathrm{HI}$ and is now a major differential diagnosis tool in infantile hyperinsulinemic hypoglycaemia. $\diamond$

\section{RéFÉRENCES}

1. Fernandes J, Saudubray JM, Van den Berghe G. Inborn metabolic diseases. Diagnosis and treatment, $3^{\text {rd }}$ ed. Heidelberg : Springer, 2001.

2. Scriver C, Beaudet A, Sly W, Valle D. The metabolic and molecular bases of inherited disease, $8^{\text {th }}$ ed. New York: MacGraw Hill, 2001.

3. Saudubray JM, Ogier $\mathrm{H}$, Bonnefont JP, et al. Clinical approach to inherited metabolic diseases in the neonatal period: a 20-year survey. J Inherit Metab Dis 1989 ; 12 (suppl 1) : 25-41.
4. Rosenberg EH, Almeida LS, Kleefstra T, et al. High prevalence of SLC6A8 deficiency in X-linked mental retardation. Am J Hum Genet 2004 ; 75 : 97-105.

5. Battini R, Leuzzi V, Carducci $C$, et al. Creatine depletion in a new case with AGAT deficiency: clinical and genetic study in a large pedigree. Mol Genet Metab 2002; 77 : 326-31.

6. Stockler S, Hanefeld F, Frahm J. Creatine replacement therapy in guanidinoacetate methyltransferase deficiency, a novel inborn error of metabolism. Lancet $1996 ; 348: 789-90$.

7. Sipila I. Inhibition of arginine-glycine amidinotransferase by ornithine. A possible mechanism for the muscular and chorioretinal atrophies in gyrate atrophy of the choroid and retina with hyperornithinemia. Biochim Biophys Acta $1980 ; 613: 79-84$.

8. Sipila I, Simell 0, Arjomaa P. Gyrate atrophy of the choroid and retina with hyperornithinemia. Deficient formation of guanidinoacetic acid from arginine. J Clin Invest $1980 ; 66: 684-7$.

9. Santinelli R, Costagliola C, Tolone C, et al. Low-protein diet and progression of retinal degeneration in gyrate atrophy of the choroid and retina : a twenty-sixyear follow-up. J Inherit Metab Dis 2004 ; 27 : 187-96.

10. Huck JH, Verhoeven NM, van Hagen JM, et al. Clinical presentations of patients with polyol abnormalities. Neuropediatrics $2004 ; 35: 167-73$.

11. Huck JH, Verhoeven NM, Struys EA, et al. Ribose-5-phosphate isomerase deficiency: new inborn error in the pentose phosphate pathway associated with a slowly progressive leukoencephalopathy. Am J Hum Genet 2004 ; 74 : 745-51.

12. Moolenaar SH, van der Knaap MS, Engelke UF, et al. In vivo and in vitro NMR spectroscopy reveal a putative novel inborn error involving polyol metabolism. NMR Biomed $2001 ; 14$ : 167-76.

13. Van der Knaap MS, Wevers RA, Struys $E A$, et al. Leukoencephalopathy associated with a disturbance in the metabolism of polyols. Ann Neurol 1999 ; $46: 925-8$.

14. Verhoeven NM, Huck JH, Roos B, et al. Transaldolase deficiency: liver cirrhosis associated with a new inborn error in the pentose phosphate pathway. Am J Hum Genet $2001 ; 68: 1086-92$.

15. Verhoeven NM, Wallot M, Huck JH, et al. A newborn with severe liver failure, cardiomyopathy and transaldolase deficiency. J Inherit Metab Dis 2005 ; 28 : 169-79.

16. Jauniaux $\varepsilon$, Hempstock J, Teng C, et al. Polyol concentrations in the fluid compartments of the human conceptus during the first trimester of pregnancy: maintenance of redox potential in a low oxygen environment. J Clin Endocrinol Metab $2005 ; 90: 1171-5$.

17. Nicol CJ, Zielenski J, Tsui LC, Wells PG. An embryoprotective role for glucose6-phosphate dehydrogenase in developmental oxidative stress and chemical teratogenesis. FASEB J $2000 ; 14$ : 111-27.

18. Yancey PH, Clark ME, Hand SC, et al. Living with water stress: evolution of osmolyte systems. Science $1982 ; 217: 1214-22$.

19. Ribeiro MJ, de Lonlay P, Delzescaux T, et al. Characterization of hyperinsulinism in infancy assessed with PET and 18F-fluoro-L-DOPA. J Nucl Med 2005; $46: 560-6$.

20. De Lonlay-Debeney P, Poggi-Travert F, Fournet JC, et al. Clinical features of 52 neonates with hyperinsulinism. N Engl J Med 1999 ; 340 : 1169-75.

21. Rahier J, Sempoux C, Fournet JC, et al. Partial or near-total pancreatectomy for persistent neonatal hyperinsulinaemic hypoglycaemia : the pathologist's role. Histopathology 1998 ; 32:15-9.

22. Sempoux C, Guiot $Y$, Jaubert F, Rahier J. Focal and diffuse forms of congenital hyperinsulinism: the keys for differential diagnosis. Endocr Pathol 2004 ; 15 : 241-6.

23. De Lonlay P, Fournet JC, Rahier J, et al. Somatic deletion of the imprinted 11 pl5 region in sporadic persistent hyperinsulinemic hypoglycemia of infancy is specific of focal adenomatous hyperplasia and endorses partial pancreatectomy. J Clin Invest 1997 ; $100: 802-7$.

24. Verkarre V, Fournet JC, de Lonlay P, et al. Paternal mutation of the sulfonylurea receptor (SUR1) gene and maternal loss of 11 pl5 imprinted genes lead to persistent hyperinsulinism in focal adenomatous hyperplasia. J Clin Invest $1998 ; 102: 1286-91$.

25. Dubois J, Brunelle F, Touati G, et al. Hyperinsulinism in children : diagnostic value of pancreatic venous sampling correlated with clinical, pathological and surgical outcome in 25 cases. Pediatr Radiol $1995 ; 25$ : 512-6.

26. Ribeiro MJ, Vidailhet M, Loc'h C, et al. Dopaminergic function and dopamine transporter binding assessed with positron emission tomography in Parkinson disease. Arch Neurol 2002 ; 59 : 580-6.

\section{TIRÉS À PART}

N. Boddaert 